\title{
Dermatofibrosarcoma Protuberans and Chronic Myeloid Leukemia: A Rare Co-incidence
}

\author{
Jhanzeb Iftikhar, Fareeha Sheikh, Nazish Khalid, Syed Abdul Mannan Hamdani, \\ Usman Ahmad, Umm-E-Kalsoom Awan
}

Department of Medical Oncology, SKMCH, Pakistan.

\begin{abstract}
Dermatofibrosarcoma protuberans (DFSP) is an uncommon, low grade, malignant fibroblastic tumor. Chronic myeloid leukemia (CML) is a myeloproliferative disorder characterized by dysregulated production of granulocytic cell line without the loss of their capacity to differentiate. To our knowledge, there is no described association of DFSP with CML in literature. The authors have encountered a first case of DFSP coexisting with chronic myeloid leukemia.
\end{abstract}

Keywords: Dermatofibrosarcoma protuberans- chronic myeloid leukemia- coexisting

Asian Pac J Cancer Care, 6 (3), 349-351

Submission Date: 01/20/2021 Acceptance Date: 05/28/2021

\section{Introduction}

Dermatofibrosarcoma protuberans (DFSP) is locally aggressive but rarely metastasizing cutaneous sarcoma [1]. It typically arises within the dermis or subcutis of the trunk, proximal extremities, or head and neck. DFSP has an indolent growth and a high tendency for local recurrences after excision [2,3]. The co-occurrence of dermatofibrosarcoma protuberans and chronic myeloid leukemia has not been reported in literature. The association is important as both DFSP (systemic disease) and chronic myeloid leukemia are responsive to tyrosine kinase inhibitor, imatinib mesylate.

\section{Case Report}

A 37-year-old male presented in oncology clinic in June 2010 with a left sided chest swelling. Magnetic resonance imaging (MRI) chest demonstrated a huge T2 hyperintense lesion in the left anterior chest wall and a hypodense lesion in the anterior mediastinum. Trucut biopsy of the left chest wall lesion showed spindle cell neoplasm (Figure 1A) was consistent with dermatofibrosarcoma protuberans (DFSP). Fine needle aspiration of anterior mediastinal mass was suggestive of benign glandular tissue. Wide local excision of chest wall lesion confirmed DFSP on histopathology. Considering DFSP a low grade category tumor and delayed graft wound healing, adjuvant radiotherapy was omitted and he was put on surveillance. He then remained well for next 3 years.

In October 2013, he landed in emergency department with a ten day history of low grade fever. Physical examination revealed palpable liver and spleen without any pallor or palpable lymph nodes. Laboratory blood profile done at that time: Complete blood count- Hemoglogin (Hb): $10.7 \mathrm{~g} / \mathrm{dL}$, platelet:325,000/ $\mu \mathrm{L}$ and white blood cell count (WBC) $212,000 / \mu \mathrm{L}$ (blast Cells 2\%, myelocytes $20 \%$, metamyelocytes $6 \%$, neutrophils $61 \%$, lymphocytes $7 \%$, monocytes $1 \%$, eosinophils $2 \%$, basophils $1 \%$ ) and markedly raised serum lactate dehydrogenase (1454 U/L). Bone Marrow aspiration and trephine biopsy (BMBx) was consistent with chronic myeloid leukemia (CML), chronic phase. Cytogenetics done on bone marrow specimen manifested GTG banding 46, XY, t (9;22) (q34;q11.2) $\{20\}$-abnormal male karyotype. All 20 GTG banded cells had a translocation between chromosomes 9 and 22 giving rise to the Philadelphia chromosome.

Hemato-oncologist started him on imatinib 400mg once a day. Three months after being on imatinib, his polymerase chain reaction, PCR BCR-ABL became below detection level and complete blood count also showed complete hematologic response with normalization of blood counts. He was continued on imatinib and his

Corresponding Author:

Dr. Jhanzeb Iftikhar

Department of Medical Oncology, SKMCH, Pakistan.

Email: jahanzaibiftikhar1986@gmail.com 


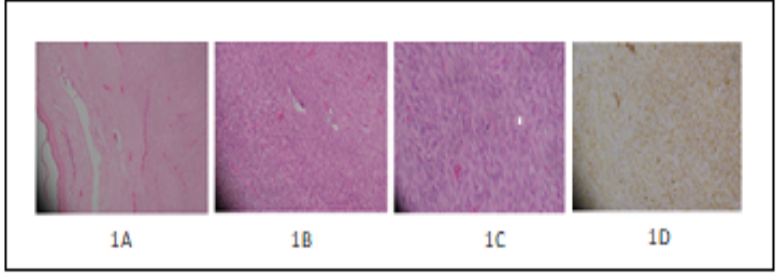

Figure 1. (A-D) Histopathological images 1A (10X magnification), Dermal Spindle Cell Tumor with Uninvolved Region between Tumor and Epidermis. 1B,C (40X magnification), Monomorphic spindle cells arranged in whirling and storiform psttern. Few typical mitoses seen. 1D (40X magnification), Diffuse CD34 positivity in tumor cells.

CML remained in major molecular remission. He was put on surveillance with 3 monthly CBC,PCR BCR-ABL and bone marrow examination. He remained well for an additional 2 years and then presented in January 2016 with a small $1.5 \times 1 \mathrm{~cm}$ fluctuant non-tender swelling on vertex. Wide local excision of right scalp lump revealed dermatofibrosarcoma protuberans on histopathology (Figure 1B,C).

After a matter of 2 more years, he again developed a subcutaneous lump in August 2018; this time it was on the right occipital area. Ultrasound guided FNA cytology of occipital lesion revealed spindle cell neoplasm. Meanwhile, he got CT thorax for symptoms of persistent cough which revealed multiple enlarged heterogenously enhancing mediastinal nodal masses along with a soft tissue mass in the anterior mediastinum infiltrating into the sternum. CT guided core biopsy of the left sided hilar mass revealed fibrosarcoma in the background of DFSP (Figure 1D). Owing to irresectability, palliative chemotherapy Ifos/Doxo (ifosphamide, doxorubicin) was planned. CT thorax after 6 cycles of chemotherapy showed decrease in the size of left hemithorax and mediastinal soft tissue masses. Recent MRI face and neck has not shown any enhancing lesion to suggest residual or recurrent disease.

From leukemia standpoint, he had been in molecular/ hematological/pathological response from the last 6 years. Imtatinib was continued considering its role both in CML and DFSP with systemic disease.

\section{Discussion}

Dermatofibrosarcoma protuberans (DFSP) presents clinically as slow growing violaceous papules, which coalesce into multinodular plaques. The most common locations are trunk and proximal extremities. Approximately $85-90 \%$ of DFSP are low grade, while the remainder contain a high-grade sarcomatous component (which is usually a fibrosarcoma designated DFSP-FS) and are considered to be intermediate-grade sarcomas [4]. By immunohistochemistry, almost all DFSP show diffuse expression of CD34 and are negative for other markers including Factor X111a and S100 protein. More than $90 \%$ of DFSP feature the characteristic reciprocal translocation, $\mathrm{t}(17 ; 22)$ (q22; $\mathrm{q} 13)$ resulting in overexpression of platelet-derived growth factor beta (PDGF $\beta 1$ ) [5]. The COL1A1-PDGF $\beta$ fusion can occasionally be cryptic and rarer rearrangements such as $\mathrm{t}(2 ; 17)$ and $\mathrm{t}(9 ; 22)$ are reported $[2,6]$.

The mainstay of therapy for DFSP is wide local excision (WLE) with tumor free margins, whereas radiotherapy is reserved for margin-positive disease where reexcision is not feasible, or for inoperable disease $[5,7]$. The recurrence potential is directly related to extent of resection $[8,9]$. DFSP is generally refractory to conventional chemotherapy. Treatment options for systemic disease are limited; however, PDGF $\beta$ R, KIT, and $\mathrm{ABL}$ inhibitor imatinib is option for systemic therapy. In published results, imatinib has shown clinical activity against localized and metastatic DFSP tumors containing t $(17 ; 22)$ (q22;q13) [3,5-9].

Chronic myeloid leukemia (CML) is associated with BCR-ABL1 fusion gene (tyrosine kinase activity) resulting from a reciprocal translocation, $t(9 ; 22)$ (q34;q11). This translocation gives rise to Philadelphia (Ph) chromosome and is found in more than $90 \%$ of the cases [10]. In the remaining cases, cryptic or complex translocations can be detected by fluorescent in-situ hybridization (FISH) or polymerase chain reaction (PCR).

To the best of our knowledge and on extensive literature research, dermatofibrosarcoma protuberans has not been reported to date in patients with CML. It is noteworthy that the lack of an identifiable link between the two neoplasms from previous studies suggests a mere coincidence in our case. As a result, it is hardly surprising that there are no references to DFSP combined with CML in literature. The question arises whether the coexistence of DFSP and CML is just a coincidence or a rare association. As both are caused by reciprocal translocation involving chromosome 22, a link between them is a strong possibility. Secondly, the pathognomic translocation in CML involves chromosome 9 and 22; very rarely a translocation involving same chromosomes, $\mathrm{t}(9 ; 22)$ is also reported in DFSP [2]. Molecular analysis of DFSP tumor using genetic studies may be helpful to see if there is a causal relationship in both these entities.

The unique coexistence of DFSP and CML is rare, but possible. Given the role of imatinib in inhibiting DFSP cell growth and its efficacy in CML, there is a need to revisit the known pathogenetic mechanisms behind DFSP and CML to speculate any possible relationship of these conditions at molecular level.

\section{References}

1. McArthur G. Dermatofibrosarcoma Protuberans: Recent Clinical Progress. Annals of Surgical Oncology. 200707 24;14(10):2876-2886. https://doi.org/10.1245/s10434-0079480-y

2. Li Y, Wang C, Xiang B, Chen S, Li L, Ji Y. Clinical Features, Pathological Findings and Treatment of Recurrent Dermatofibrosarcoma Protuberans. Journal of Cancer. 2017;8(7):1319-1323. https://doi.org/10.7150/jca.17988

3. Molina AS, Duprat Neto JP, Bertolli E, da Cunha IW, Fregnani JH, Figueiredo PH, Soares FA, Macedo MP, Pinto Lopes 
CA, de Abranches Oliveira Santos Filho ID. Relapse in dermatofibrosarcoma protuberans: A histological and molecular analysis. Journal of Surgical Oncology. 2018 03 06;117(5):845-850. https://doi.org/10.1002/jso.25039

4. Buck DW, Kim JY, Alam M, Rawlani V, Johnson S, Connor CM, Dumanian GA, Wayne JD. Multidisciplinary approach to the management of dermatofibrosarcoma protuberans. Journal of the American Academy of Dermatology. 2012 Nov;67(5):861-866. https://doi.org/10.1016/j. jaad.2012.01.039

5. Thway K, Noujaim J, Jones RL, Fisher C. Dermatofibrosarcoma protuberans: pathology, genetics, and potential therapeutic strategies. Annals of Diagnostic Pathology. 2016 Dec;25:6471. https://doi.org/10.1016/j.anndiagpath.2016.09.013

6. Sonobe H, Furihata M, Iwata J, Ohtsuki Y, Chikazawa M, Taguchi T, Shimizu K. Dermatofibrosarcoma Protuberans Harboring $\mathrm{t}(9 ; 22)(\mathrm{q} 32 ; \mathrm{q} 12.2)$. Cancer Genetics and Cytogenetics. 1999 04;110(1):14-18. https://doi.org/10.1016/ s0165-4608(98)00183-6

7. Rutkowski P, Debiec-Rychter M. Current treatment options for dermatofibrosarcoma protuberans. Expert Review of Anticancer Therapy. 201505 31;15(8):901-909. https://doi. org/10.1586/14737140.2015.1052799

8. Woo K, Bang SI, Mun G, Oh KS, Pyon J, Lim SY. Long-term outcomes of surgical treatment for dermatofibrosarcoma protuberans according to width of gross resection margin. Journal of Plastic, Reconstructive \& Aesthetic Surgery. 2016 03;69(3):395-401. https://doi.org/10.1016/j. bjps.2015.10.027

9. Rutkowski P, Van Glabbeke M, Rankin CJ, Ruka W, Rubin BP, Debiec-Rychter M, Lazar A, Gelderblom H, Sciot R, LopezTerrada D, Hohenberger P, van Oosterom AT, Schuetze SM. Imatinib Mesylate in Advanced Dermatofibrosarcoma Protuberans: Pooled Analysis of Two Phase II Clinical Trials. Journal of Clinical Oncology. 201004 01;28(10):1772-1779. https://doi.org/10.1200/jco.2009.25.7899

10. Groffen J, Stephenson J, Heisterkamp N, DeKlein A, Bartram C, Grosveld G. Philadelphia chromosomal breakpoints are clustered within a limited region, bcr, on chromosome 22 . Cell. 1984 01;36(1):93-99. https://doi.org/10.1016/00928674(84)90077-1

\section{(ब) 0 (8)}

This work is licensed under a Creative Commons AttributionNon Commercial 4.0 International License. 